\title{
Flexible Numerology in 5G NR: Interference Quantification and Proper Selection Depending on the Scenario
}

\author{
Josue Flores de Valgas $\mathbb{D},{ }^{1}$ Jose F. Monserrat $\mathbb{D}^{1},{ }^{1}$ and Hüseyin Arslan $\mathbb{D D}^{2}$ \\ ${ }^{1}$ Universitat Politècnica de València, iTEAM Research Institute, Camino de Vera s/n, 46022 Valencia, Spain \\ ${ }^{2}$ Department of Electrical and Electronics Engineering, Istanbul Medipol University, Istanbul 34810, Turkey \\ Correspondence should be addressed to Josue Flores de Valgas; joflode1@teleco.upv.es
}

Received 15 October 2020; Revised 1 February 2021; Accepted 1 March 2021; Published 10 March 2021

Academic Editor: Massimo Condoluci

Copyright ( 2021 Josue Flores de Valgas et al. This is an open access article distributed under the Creative Commons Attribution License, which permits unrestricted use, distribution, and reproduction in any medium, provided the original work is properly cited.

\begin{abstract}
The 3rd Generation Partnership Project (3GPP) adopted cyclic prefix OFDM (CP-OFDM) for both uplink and downlink communications (although DFT-s-OFDM is also allowed in the uplink) in 5G New Radio (NR) Release 15. However, due to the variety of proposed deployment options and scenarios, a single numerology will not be enough to fulfil all performance requirements. A scalable OFDM numerology was required to enable diverse services on a wide range of frequencies and deployments, and finding the right numerology for each scenario is of special relevance for the proper functioning of $5 \mathrm{G}$ NR. Using a simulator calibrated according to the parameters established for NR performance by the 3GPP, this paper presents the performance evaluation of NR for the main $5 \mathrm{G}$ scenarios and different CP-OFDM numerologies and device speeds. Results show that increasing subcarrier spacing boosts the strength of the system against intercarrier interference (ICI) caused my Doppler spread; however, to increase subcarrier spacing, the CP must be reduced proportionally, which makes intersymbol interference (ISI) and ICI caused by insufficient CP have a more predominant effect. Therefore, it is necessary to quantify the total interference of the system, in order to determine the proper numerology for each scenario, which will depend on all the factors mentioned above, and not only on the operation band, as suggested in the standardization process. All this allows concluding that the choice of the appropriate numerology for a particular system depends not only on the band of operation but also on the deployment scenario and the speed of the user equipment (UE). Likewise, it is concluded that it is even possible to use more than one numerology for the same scenario.
\end{abstract}

\section{Introduction}

Since the beginning of 2016, the 3rd Generation Partnership Project (3GPP) has been working on the standardization of 5G New Radio (NR) [1], a new Radio Access Technology (RAT) that will guarantee the performance, interoperability, and quality of $5 \mathrm{G}$ devices and networks, within next-generation global standard [2]. This will allow services such as virtual reality, augmented reality, automated intelligence, autonomous vehicles, and the Internet of Things (IoT) [3] to become a reality. In order to provide the aforementioned services, a new generation of mobile communication systems will be necessary since these are based on more reliable, more prompt, and even faster interconnectivity.

To meet all this variety of services, which $5 \mathrm{G}$ standard is designed to satisfy, there are three main challenges that $5 \mathrm{G}$
NR must solve in order to enable a truly networked society: a higher data rate, more reliable and low latency transmissions, and a massive growth in the number of devices. These challenges result in three broad use cases [4]: enhanced mobile broadband (eMBB), which requires very high data rates and large bandwidths, e.g., highly mobile UE connected to macrocells; ultra-reliable low-latency communications (uRLLC), which require very high reliability and availability, as very low latency, e.g., power system automation and factory process; and massive machine type communications (mMTC), which require low energy consumption at the UE, high connection density, and low bandwidth, e.g., collection of the measurements from a massive number of sensors.

Several candidate waveforms were submitted to the $5 \mathrm{G}$ NR standardization process, many of which had been under study even before the formal beginning of this new 
specification [5-7]. Most of these candidates are multicarrier waveforms, like the well-known cyclic prefix OFDM (CPOFDM), universally filtered OFDM (also known as universal filtered multicarrier (UFMC)), pulse shaped OFDM (POFDM), windowed OFDM (W-OFDM), and filter-bank multicarrier (FBMC). Nevertheless, after multiple discussions and a long evaluation process, in August 2016, the 3GPP decided to utilize CP-OFDM for both downlink and uplink in NR Release 15. Being built over OFDM (which is used on LTE) gives 5G NR an advantage since it allows the devices to keep low complexity and hence low hardware costs. However, a single OFDM numerology, i.e., subcarrier spacing and cyclic prefix length, cannot satisfy the performance constraints across the desired frequency range and all proposed deployment options and scenarios. This is why the OFDM numerology must be adapted to fit the specific requirements of services, operation frequencies, and deployment scenarios.

This paper provides an in-depth analysis of the use of $5 \mathrm{G}$ numerologies within the main $5 \mathrm{G}$ scenarios, using TDL-C channel model and taking into account the operating frequency and the mobility of the UE. Although there are some studies regarding OFDM numerology itself $[8,9]$ and some other approaches considering the use of multiple numerologies at the same time, looking for the lowest internumerology interference (INI) [10-12], there is currently no evaluation of the impact of numerology changes on the total system interference, making possible to determine whether a numerology is appropriate for a particular 5G scenario. This paper's main contribution is to demonstrate that it is possible to use more than a single 5G OFDM numerology for every proposed band throughout the $5 \mathrm{G}$ spectrum. In order to determine which numerology is better suitable for each scenario, the total interference of the system was calculated for all possible scenarios, taking into account the topology, operating frequency, and mobility, allowing to determine which numerology is better fitted for each scenario. The remaining sections of the paper are structured as follows. Section 2 describes the analysis of interference calculation, while Section 3 provides an explanation of the 5G OFDM numerologies. Section 4 presents the performance results. Finally, Section 5 draws the main conclusions of this work.

\section{ICI and ISI Analysis}

In an OFDM system, the transmitted data are mapped to some constellation points in order to obtain data symbols. The transmitter then applies an $\mathrm{N}$-point inverse discrete Fourier transform (IDFT), with the purpose of obtaining the time-domain OFDM symbols. The time-domain OFDM $n$ th block $x[n]=\left[x_{n, 0}, x_{n, 1}, \cdots, x_{n, N-1}\right]$ is given by

$$
x[n]=F_{N}^{H} S[n],
$$

where $F_{N}^{H}$ is the $N$-point IDFT matrix, $S[n]=\left[s_{n, 0}, s_{n, 1}, \cdots\right.$, $\left.s_{n, N-1}\right]$ is the transmitted symbol, and $N$ is the block size. After the IDFT block, the cyclic prefix (CP) is added, which is a copy of the last $M$ samples of the IDFT output. The main function of the $\mathrm{CP}$ is to avoid the overlapping between consecutive OFDM symbols, but it also turns the linear convolution by the channel into circular, thus allowing to use frequency-domain subcarrier-wise equalizer. In the receiver side, the $\mathrm{CP}$ is discarded, and then an $\mathrm{N}$-point FFT is applied. In the absence of noise, the $n$-th received block $\widehat{X}[n]=\left[\widehat{X}_{n, 0}, \widehat{X}_{n, 1}, \cdots, \widehat{X}_{n, N-1}\right]$ is

$$
\widehat{X}[n]=H[n] x[n],
$$

where $H[n]$ is the time-domain channel matrix, formed by the elements of the channel impulse response during the $n$ th block interval. This OFDM structure can be affected by several external aspects, which trigger harmful effects on the system's performance by modifying the structure in a disruptive manner; this is what is known as interference. In the case of a scenario with insufficient CP, the system is affected by intersymbol interference (ISI) and intercarrier interference (ICI), whereas in a scenario with a high Doppler spread, the system is also affected by ICI.

2.1. Insufficient Cyclic Prefix. In the presence of a multipath channel, what happens is that at the receiver side, all the multipath components are summed. As a result, we have multiple echoes of each symbol arriving at multiple instances of time and overlapping with each other, causing data to get corrupted and consequently lost. The total delay between the first echo of the symbol and the last one can be measured through the delay spread (DS) concept. Therefore, most of the energy caused by the distortion in the symbol reaches up to certain DS given by the characteristic of the channel.

If the DS is longer than the CP (DS > CP), then the echoes will arrive during the core OFDM symbol, and there will be a certain percentage of interference in the signal. Therefore, by extending the CP length in such a way that it is longer than the DS (CP > DS), the overlap will happen only during the $\mathrm{CP}$, which is discarded at the receiver, and hence there will not be interference due to the DS.

One of the main benefits of using a CP between consecutive OFDM symbols is that it isolates them from each other, acting as a guard interval to protect the OFDM signal from ISI. However, there are cases where the CP can be shorter than the DS, which causes ISI because of the overlapping of subsequent symbols. On the other hand, a shorter OFDM symbol period makes subcarriers more separated from each other, which at the end is good to combat ICI. There is a compromise between counteracting the effects of ISI and suffering from more effects of ICI. This can be introduced by extending the model in equation (2). Considering a channel with an impulse response of length $L$ and a CP of length $M$, if $L>M$, then the $n$-th received block now would be [13]

$$
\widehat{X}[n]=H[n] x[n]+\widehat{X}_{1}[n]+\widehat{X}_{2}[n],
$$

where $\hat{X}_{1}[n]=B X^{(i-1)}$ and $\hat{X}_{2}[n]=-A X^{(i)} ; X^{(i-1)}$ is the previous transmitted symbol; $X^{(i)}$ is the current transmitted symbol; $B$ is the $N \times N$ contribution of the elements of the channel impulse response that exceeds the $\mathrm{CP}$, as seen in equation (5); and $A$ is the same matrix as $B$ but with 
circularly shifted $L-M$ columns to the left, as seen in the following equation:

$$
\begin{aligned}
A & =\left[\begin{array}{ccccccccc}
0 & \cdots & h_{M} & h_{M-1} & \cdots & h_{L+1} & 0 & \cdots & 0 \\
0 & \cdots & 0 & h_{M} & \cdots & h_{L+2} & 0 & \cdots & 0 \\
\vdots & \cdots & \vdots & \ddots & \ddots & \vdots & \vdots & \vdots & \vdots \\
0 & \cdots & 0 & 0 & \ddots & h_{M} & 0 & \cdots & 0 \\
\vdots & \cdots & \vdots & \ddots & \ddots & \vdots & \vdots & \vdots & \vdots \\
0 & \cdots & 0 & 0 & \cdots & 0 & 0 & \cdots & 0
\end{array}\right], \\
B & =\left[\begin{array}{cccccccc}
0 & \cdots & 0 & \cdots & h_{M} & \cdots & \cdots & h_{L+1} \\
0 & \cdots & 0 & \cdots & 0 & h_{M} & \cdots & h_{L+2} \\
\vdots & \cdots & \vdots & \ddots & \ddots & \vdots & \vdots & \vdots \\
0 & \cdots & 0 & \ddots & \ddots & 0 & \cdots & h_{M} \\
\vdots & \cdots & \vdots & \ddots & \ddots & \vdots & \vdots & \vdots \\
0 & \cdots & 0 & \cdots & 0 & \cdots & \cdots & 0
\end{array}\right] .
\end{aligned}
$$

In Figure 1(a), we can see how the previous OFDM symbol $\widehat{X}[n-1]$ overlaps a portion of the current symbol $\widehat{X}[n]$; this is known as ISI and would correspond to $\widehat{X}_{1}[n]$ in equation (3). Likewise, it can be seen that the last path contains a part of the current symbol that interferes with itself. This can be considered as a subcarrier shift since it is the same symbol, and therefore it is possible to refer to this as ICI, thus corresponding with the term $\widehat{X}_{2}[n]$ in equation (3). Figure 1(b) shows that when the CP is greater than the DS, there is no ISI or ICI.

2.2. Doppler Spread. The mobility of the user equipment with respect to the receiver, that is, the base station, produces a variation in the incoming received signal. This is known as Doppler spread and is explained due to the frequency shift that each multipath component experiences as it propagates from the transmitter to the receiver. The rate of this shift (Doppler shift) depends on the speed and direction of the mobile terminal (although in 5G the use of mobile base stations could be expected). The frequency of the received signal will increase as it travels over the air, as long as the distance between the transmitter and receiver decreases; conversely, it will decrease when the distance increases.

The above can be explained taking into account the following [14]. Assuming that the user equipment is moving at constant speed $v$, at the receiver side each multipath echo will arrive at different instant of time, different phase, and different amplitude. The phase change by a single multipath echo in the received signal, due to difference in path lengths, is given by

$$
\Delta \phi=\frac{2 \pi v \Delta t}{\lambda} \cos \theta
$$

where $\lambda=c / f_{c}$ is the carrier wavelength, $f_{c}$ is the carrier frequency, $c$ is the speed of light, $\Delta t$ is the time difference between the arrival of the previous multipath echo and the current one, and $\theta$ is the angle of arrival of the current multipath echo. The Doppler shift is defined as the rate of phase change due to moving; therefore, using the terms in equation (6), it can be derived as

$$
f=\frac{1}{2 \pi} \cdot \frac{\Delta \phi}{\Delta t}=\frac{\nu f_{c}}{c} \cos \theta .
$$

The maximum Doppler shift occurs when the transmitter and the receiver are moving in opposite or same direction $(\theta=\pi$ or $2 \pi)$ :

$$
f_{\max }=\frac{v f_{c}}{c}
$$

As said before, Doppler shift is the effect caused by a single multipath echo, which typically occurs in a free space scenario with line-of-sight (LOS). Doppler spread, instead, is a random effect caused by several multipath components coming from different directions. In both cases, ICI affects the system, but the one produced by Doppler spread is harder to deal with because it can degrade considerably the performance of OFDM systems since subcarriers while shifting lose the required orthogonality with their neighbors. An exact equation for the ICI power created by Doppler spread is derived in [15] and is given as

$$
P_{\text {ICI }}=\frac{\left(f_{\max } T_{S}\right)^{2}}{2} \sum_{\substack{k=1 \\ k \neq i}}^{N} \frac{1}{(k-i)^{2}},
$$

where $i$ represents the subcarrier on which the interference is measured and $k$ represents all other subcarriers of the OFDM symbol.

2.3. Unified Interference Model. In [13], a unified interference system model is proposed. In the case of a doubly selective channel, the channel impulse response is timevariant during an OFDM block, so the first term in equation (3) can be rewritten as the sum of two components:

$$
\widehat{X}[n]=\widehat{X}_{1}[n]+\widehat{X}_{2}[n]+\widehat{X}_{3}[n]+\widehat{X}_{4}[n],
$$

where

$$
\begin{aligned}
& \widehat{X}_{3}[n]=H_{\mathrm{ave}}^{[n]} x[n], \\
& \widehat{X}_{4}[n]=H_{\mathrm{var}}^{[n]} x[n],
\end{aligned}
$$

where $H_{\text {ave }}^{(i)}$ is the time average channel impulse response matrix for the $i$-th received block and $H_{\text {var }}^{(i)}$ represents the channel variation from the average. The model proposes that $\widehat{X}_{3}[n]$ is the ICI-free and ISI-free term, while $\widehat{X}_{4}[n]$ represents the ICI caused by the channel variations and corresponds to the equation specified in equation (9), i.e., the ICI power produced by Doppler spread.

\section{5G NR Numerology}

As previously stated, OFDM plays a very important role in $5 \mathrm{G} \mathrm{NR}$; nonetheless, to meet all the specifications that are necessary within the new $5 \mathrm{G}$ landscape, more than a single 


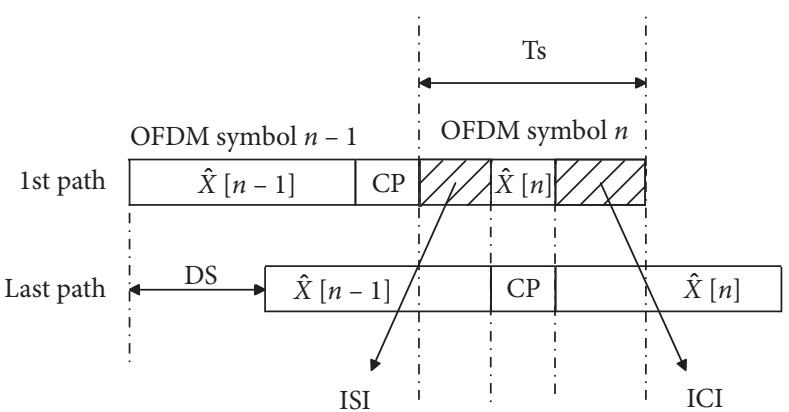

(a)

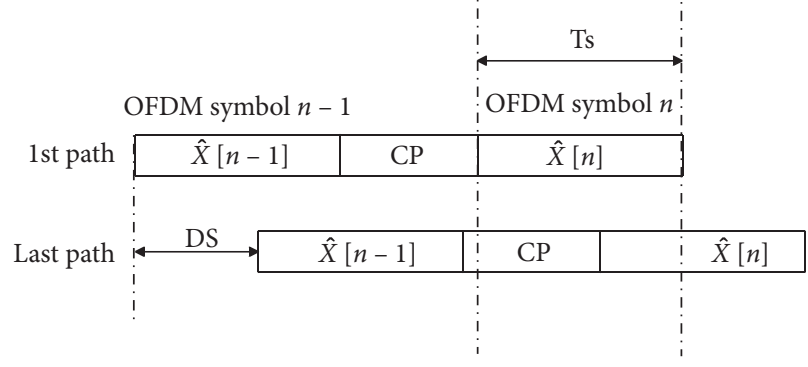

(b)

FIGURE 1: Relationship between the DS and CP and its effect on the ICI and ISI. (a) Insufficient CP scenario with the presence of ISI and ICI. (b) Scenario with CP larger than DS and no ISI or ICI.

fixed OFDM numerology is needed. In 4G, LTE supports carrier bandwidths up to $20 \mathrm{MHz}$ with an established OFDM numerology, i.e., a fixed $\mathrm{CP}$ duration $\left(T_{\mathrm{CP}}\right)$ and a fixed subcarrier spacing $(\Delta f)$; more precisely, LTE uses a $4.69 \mu \mathrm{s} \mathrm{CP}$ and a $15 \mathrm{kHz}$ subcarrier spacing. On the other hand, for $5 \mathrm{G} \mathrm{NR}$, the purpose is to introduce scalable numerology OFDM with the aim of supporting various scenarios, deployment models, and a wide range of frequencies. One of the most critical requirements is that the OFDM subcarrier spacing must be able to scale with the channel bandwidth, so the processing complexity does not increase exponentially for wider bandwidths, as the FFT size scales.

Consequently, the main difference between $5 \mathrm{G}$ NR and LTE frame structure is that the first can support different numerologies, which implies changes in the subcarrier spacing, CP length, symbol duration, etc. The parameter that sets the changes in the numerology is the subcarrier spacing, and it can be scaled according to the following factor: $15 \times 2^{n} \mathrm{kHz}$, where $n$ is an integer and $15 \mathrm{kHz}$ is the subcarrier spacing used in LTE. By using this $2^{n}$ factor, 5G NR ensures that slots and symbols of different numerologies are aligned in the time domain, which is important to efficiently enable time division duplex (TDD) networks.

There are a variety of factors that influence the suitability of a particular numerology for a given scenario, including mobility, service requirements (latency, reliability, and throughput), type of deployment, carrier frequency, and implementation complexity. For instance, wider subcarrier spacing can be more appropriated for small coverage areas, latency-critical uRLLC services, and higher carrier frequencies, which could be the case of a V2X scenario with high mobility. A graphic explanation of the different channel widths and different scalable deployment types can be seen in Figure 2. Finally, there is the number of OFDM symbols within a slot, which despite not changing intrinsically when changing the numerology is necessarily adjusted so that the time alignment is not lost. For any numerology, it will always be 14 (except for the $60 \mathrm{kHz}$ subcarrier spacing with extended CP case, which uses 12 symbols per slot), unlike LTE that had two slots with 7 symbols each. The summary of 5G NR numerology can be seen in Table 1 .

\section{Performance Results}

The performance comparison of the different 5G NR numerologies at various speeds and frequencies and within most common 5G scenarios was made with 1000 transmission time intervals (TTI) per numerology, using the PHY layer parameters of LTE and adjusting the frame structure with the parameters of the numerologies shown in Table 1, a 64-QAM modulation, and a tapped delay line- (TDL-) C 300 [16] channel model with 24 taps. The chosen speeds were 3, 120 , and $300 \mathrm{~km} / \mathrm{h}$, in order to simulate low, medium, and high mobility scenarios. Regarding the frequency of operation, the band from $400 \mathrm{MHz}$ to $6 \mathrm{GHz}$ was used (spectrum utilized by LTE), as well as frequencies higher than $6 \mathrm{GHz}$, which are intended to be used in $5 \mathrm{G} \mathrm{NR}$, especially those that correspond to millimeter waves (mmWave). Finally, based on an LTE PHY layer simulator, calibrated according to the parameters established for NR performance reference in the 3GPP RAN1\#85 meeting [17], changes were made to the CP and subcarrier spacing, in order to obtain the level of interference for every possible scenario.

The TDL-C 300 channel is constructed to represent a particular channel profile for NLOS, allowing to obtain a desired RMS delay spread for a specific scenario by scaling every tap delay according to the following equation:

$$
\tau_{n, \text { scaled }}=\tau_{n, \text { model }} \cdot \mathrm{DS}_{\text {desired }}
$$

where $\tau_{n \text {,model }}$ is the normalized delay value of the $n$-th path, $\tau_{n, \text { scaled }}$ is the new delay value in $[n s]$, and $\mathrm{DS}_{\text {desired }}$ is the wanted delay spread in $[n s]$.

The TDL-C 300 model can be scaled to other channel scenarios optimized for $5 \mathrm{G} \mathrm{NR}$, that is, for frequencies up to $100 \mathrm{GHz}$. This is achieved thanks to a scaling factor, which allows adapting the delays for each scenario. The scenarios used are Indoor office, Urban Micro Street-canyon (UMi Street-canyon), Urban Macro (UMa), Rural Macro (RMa), Urban Micro (UMi), and Urban Macro Outdoor-to-Indoor (UMa O2I) [18].

Table 2 [16] shows different scaling factors, which have been chosen in such a way that the RMS delay spread covers the entire range observed in the measurements corresponding to the proposed $5 \mathrm{G}$ scenarios. The values of "normal-delay profile" and "long-delay profile" correspond 


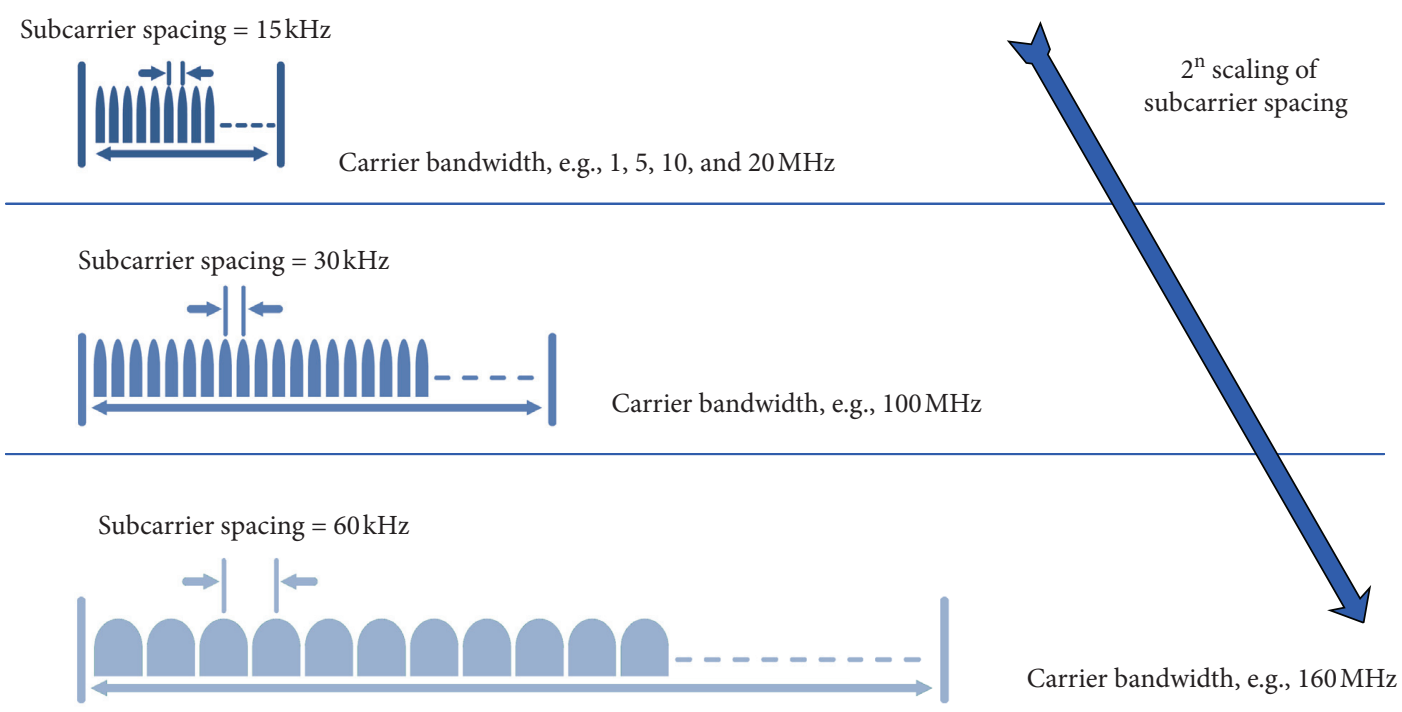

Figure 2: Example channel bandwidths and subcarrier spacing.

TABLE 1: Numerology structures for 5G NR.

\begin{tabular}{lccccc}
\hline$\Delta f=15 \times 2^{n}(\mathrm{kHz})$ & Symbol duration $(\mu \mathrm{s})$ & $\mathrm{T}_{\mathrm{CP}}(\mu \mathrm{s})$ & Slot duration $(\mathrm{ms})$ & Number of slots/subframes & Number of symbols/slots \\
\hline 15 & 66.67 & 4.69 & 1 & 1 & 14 \\
30 & 33.33 & 2.34 & 0.5 & 2 & 14 \\
60 & 16.67 & 1.17 & 0.25 & 4 & 14 \\
120 & 8.33 & 0.57 & 0.125 & 8 & 14 \\
\hline
\end{tabular}

TABLE 2: Scenario-specific scaling factors.

\begin{tabular}{|c|c|c|c|c|c|c|c|c|}
\hline \multirow{2}{*}{ Proposed scaling factor } & & \multicolumn{7}{|c|}{ Frequency $(\mathrm{GHz})$} \\
\hline & & 2 & 6 & 15 & 28 & 39 & 60 & 70 \\
\hline \multirow{2}{*}{ Indoor office } & Normal-delay profile & 39 & 30 & 24 & 20 & 18 & 16 & 16 \\
\hline & Long-delay profile & 59 & 53 & 47 & 43 & 41 & 38 & 37 \\
\hline \multirow{2}{*}{ UMi Street-canyon } & Normal-delay profile & 129 & 93 & 76 & 66 & 61 & 55 & 53 \\
\hline & Long-delay profile & 634 & 316 & 307 & 301 & 297 & 293 & 291 \\
\hline \multirow{2}{*}{ UMa } & Normal-delay profile & 363 & 363 & 302 & 266 & 249 & 228 & 221 \\
\hline & Long-delay profile & 1148 & 1148 & 955 & 841 & 786 & 720 & 698 \\
\hline \multirow{2}{*}{$\mathrm{RMa}$} & Normal-delay profile & 37 & 37 & N/A & N/A & N/A & N/A & N/A \\
\hline & Long-delay profile & 153 & 153 & N/A & N/A & N/A & N/A & N/A \\
\hline \multirow{2}{*}{ UMi/UMa O2I } & Normal-delay profile & & & & 240 & & & \\
\hline & Long-delay profile & & & & 616 & & & \\
\hline
\end{tabular}

to the median and 90th percentile RMS delay spread for NLOS scenarios.

These "normal-delay profile" and "long-delay profile" DS values are obtained through several measurements and estimations made for the different scenarios used. It can be seen that the highest values of DS are those of the UMa scenario, and this is due to the fact that in this scenario, more objects are present, which causes more echoes to occur and therefore increases the DS. Something similar happens with the UMi/Uma O2I and UMi Street-canyon scenarios; however, the latter has the peculiarity that it confines the signal as if it were a corridor, and due to this topology, it presents a lower DS than the other urban scenarios. Finally, we have the Indoor office and RMa scenarios, which are the ones with the lowest DS of all the proposed scenarios; in the case of the first one, it is due to its small coverage area topology, with many objects that reflect the signal, but all of them relatively close to the receiver, while in the case of the latter, it is a topology with open spaces, so there are few objects that produce echoes, and therefore the DS is low. All these topologies are visually represented in Figure 3 for the sake of clarity and intuitive explanation.

After carrying out the simulations with the different configurations, it was possible to measure the percentage of interference in every scenario. For this, the model in (10) was taken as a reference, and from it the power of $\hat{X}[n]$ was normalized, and the power of the components $\widehat{X}_{1}[n], \widehat{X}_{2}[n]$, and $\widehat{X}_{4}[n]$ was calculated. Consequently, the sum of the power of $\widehat{X}_{1}[n]$ and $\widehat{X}_{2}[n]$ would be the percentage of interference due to insufficient cyclic prefix, and the power of 


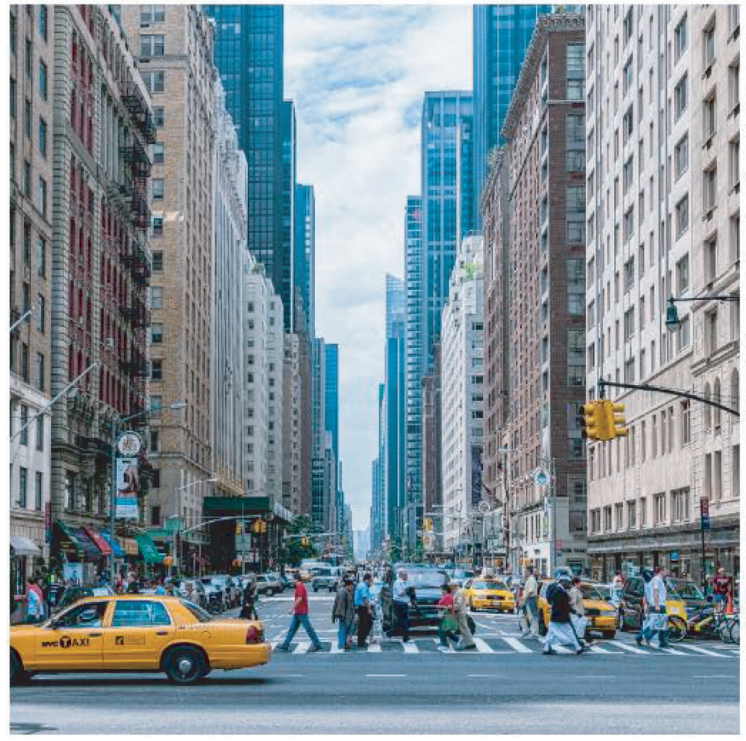

UMi street-canyon

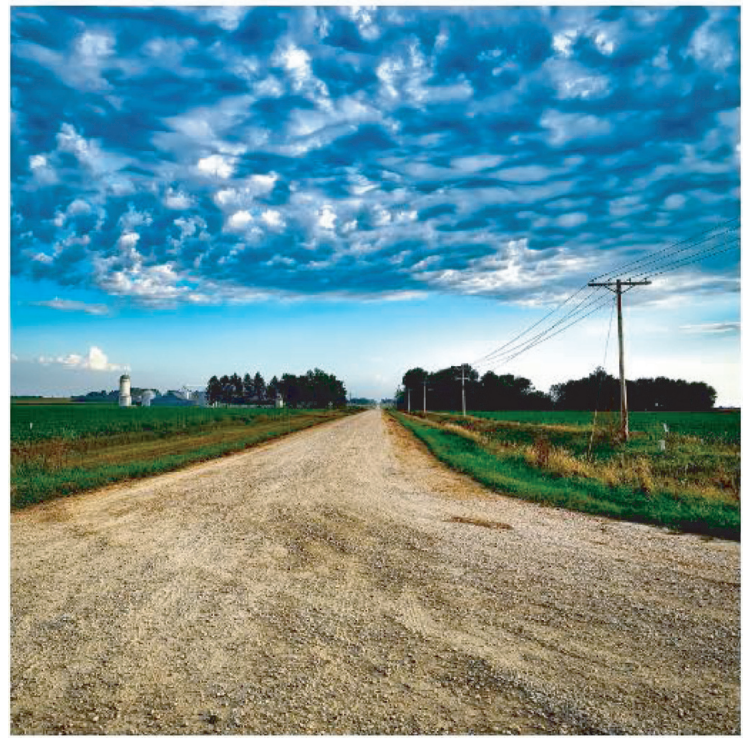

$\mathrm{RMa}$

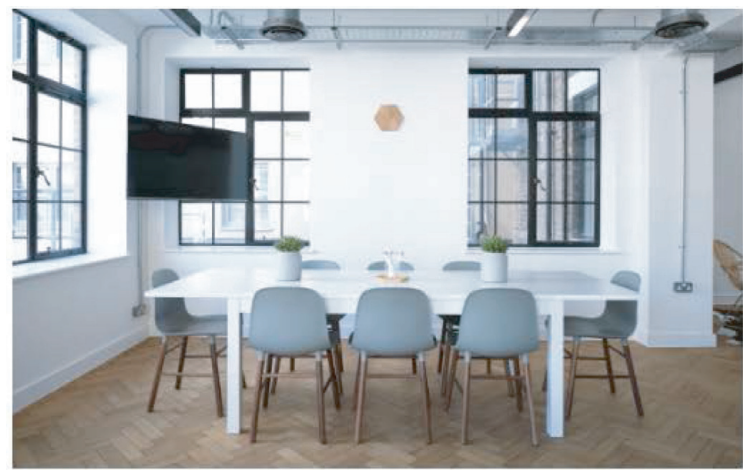

Indoor office

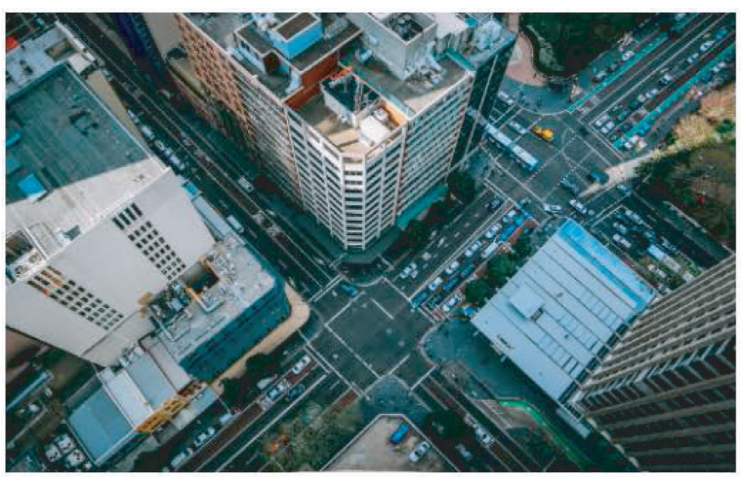

$\mathrm{UMa}$

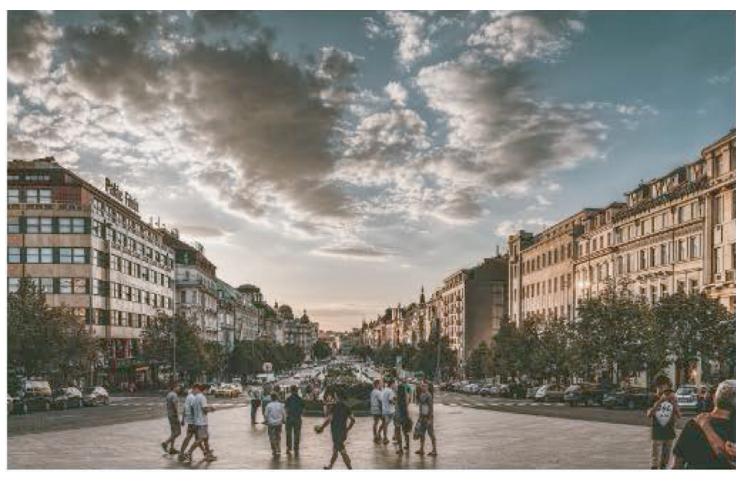

UMi/UMa 021

Figure 3: Model scenario topology examples.

$\widehat{X}_{4}[n]$ would be the percentage of interference due to the Doppler spread.

The results corroborated what was previously explained, i.e., when increasing the speed, the ICI caused by the Doppler spread also increases, while changing numerology and increasing the subcarrier spacing, the CP is reduced and the signal becomes more prone to the ISI and ICI caused by insufficient CP. By adding up the interference of both effects, it is possible to obtain the total percentage of interference of the system for each different scenario. In Figure 4 , it is possible to observe a comparison between the interference caused by the Doppler spread; the interference caused by insufficient CP; and the total interference of an UMa scenario at $120 \mathrm{~km} / \mathrm{h}$. In the graph of the interference caused by Doppler spread, it can be seen how for the cases of $15 \mathrm{kHz}$ and $30 \mathrm{kHz}$ subcarrier spacing, the interference increases as the frequency increases, while for $60 \mathrm{kHz}$ and $120 \mathrm{kHz}$ subcarrier spacing, there is hardly an increase in the interference. On the other hand, in the graph of interference caused by insufficient $\mathrm{CP}$, it can be appreciated that for a $120 \mathrm{kHz}$ subcarrier spacing interference, the DS is so large that the echoes completely overlap the next symbol; therefore, this would mean a $100 \%$ ISI case. Finally, by considering both interferences, it is possible to determine the best numerology according to the band in use.

Figure 5 shows which numerology can be used according to the operation frequency, speed, and topology. The first thing to note is that for frequencies $<3 \mathrm{GHz}$, the results show that the $15 \mathrm{kHz}$ subcarrier spacing is the best option and still a valid option for frequencies between $3 \mathrm{GHz}$ and $6 \mathrm{GHz}$. 


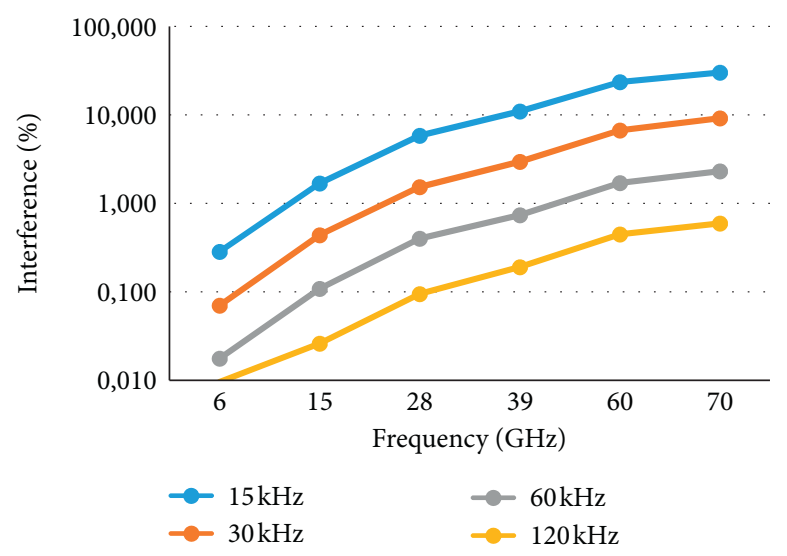

(a)

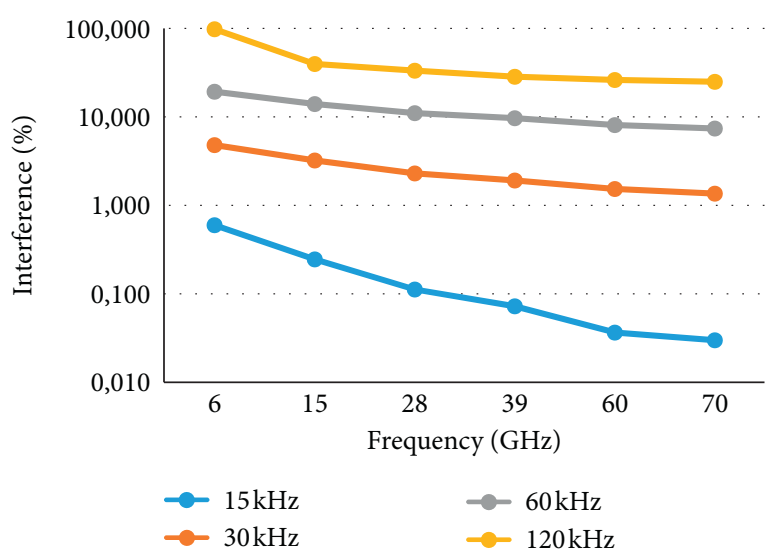

(b)

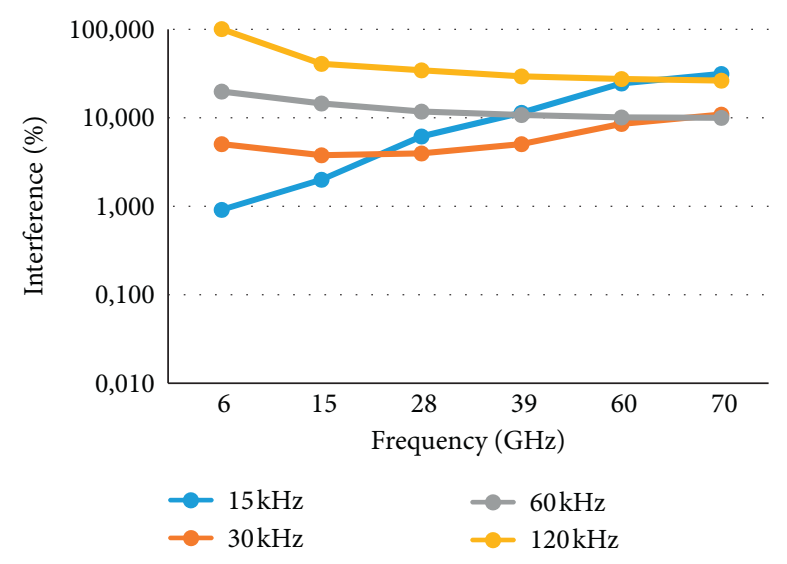

(c)

FIGURE 4: Interference comparison for an UMa scenario at $120 \mathrm{~km} / \mathrm{h}$. (a) Doppler interference. (b) Insufficient CP interference. (c) Total interference.

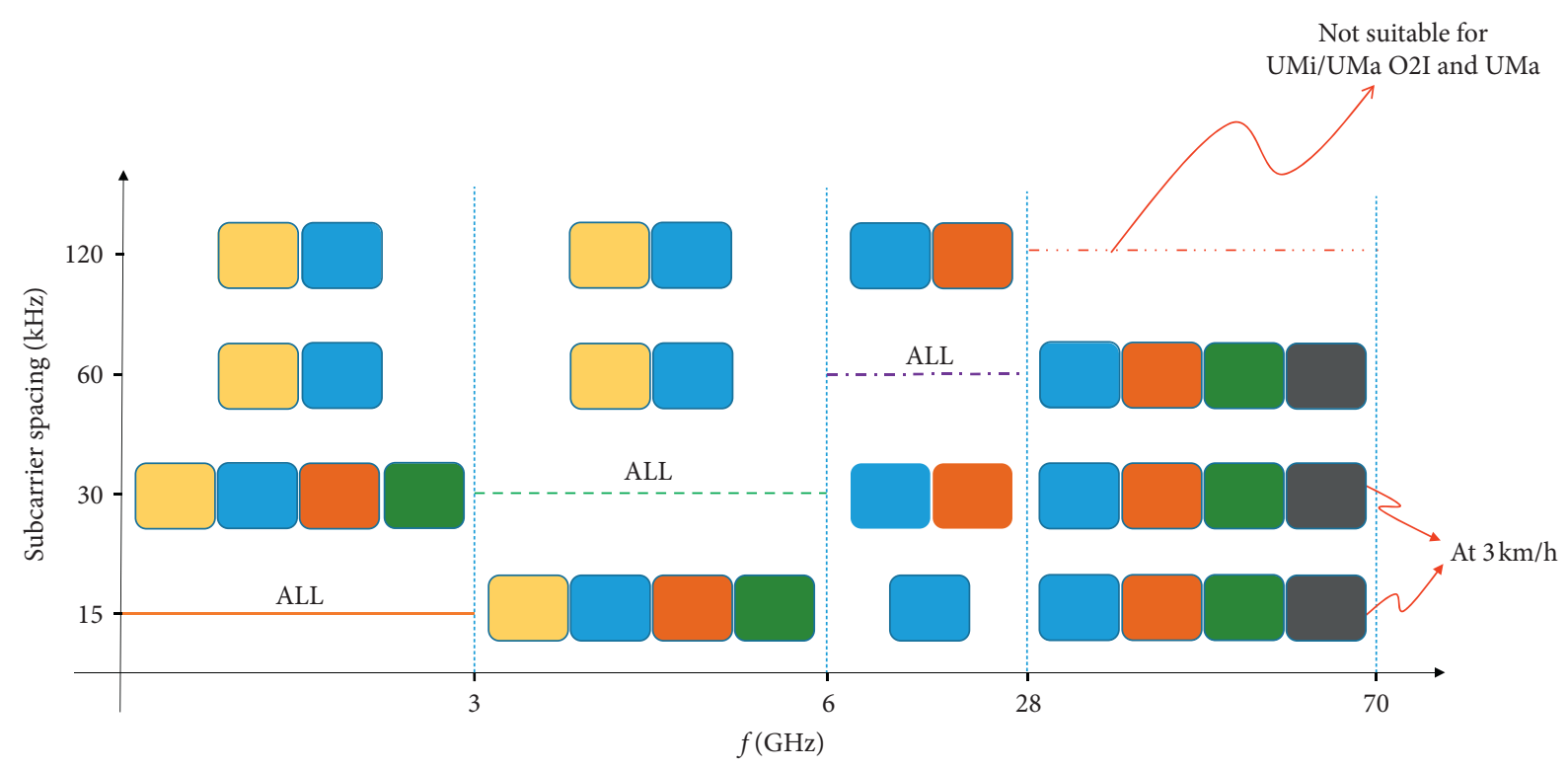

$\mathrm{RMa}$

UMi/UMa O2I

Indoor office

$\mathrm{UMa}$

UMi street-canyon

FIgURE 5: Allowed numerologies for each 5G scenario. 
This is something to be expected since it is the spectrum used for LTE, which corroborates that $4 \mathrm{G}$ systems are currently working optimally. However, for the $<3 \mathrm{GHz}$ band, it is possible to use different subcarrier spacing; i.e., $30 \mathrm{kHz}$ subcarrier spacing can be used in UMi Street-canyon, Indoor office, RMa, and UMi/Uma O2I environments, while $60 \mathrm{kHz}$ and $120 \mathrm{kHz}$ subcarrier spacings can be used in Indoor office and $\mathrm{RMa}$ environments. Regarding the $3-6 \mathrm{GHz}$ band, where the recommendation is to use a $30 \mathrm{kHz}$ subcarrier spacing, Figure 4 shows that as in the $<3 \mathrm{GHz}$ band, it is possible to use a $15 \mathrm{kHz}$ subcarrier spacing in UMi Street-canyon, Indoor office, RMa, and $\mathrm{UMi} / \mathrm{Uma} \mathrm{O} 2 \mathrm{I}$ environments and also $60 \mathrm{kHz}$ and $120 \mathrm{kHz}$ subcarrier spacings in Indoor office and RMa environments. An interesting detail is that for this range of frequencies (3-6 GHz), in both Indoor office environment and RMa environment, it is possible to use any numerology since the total interference does not exceed $1 \%$.

For frequencies between 6 and $28 \mathrm{GHz}$ (before mmWave), the recommendation is to use a $60 \mathrm{kHz}$ subcarrier spacing, but as shown in Figure 5, it is possible to use a $15 \mathrm{kHz}$ subcarrier spacing in the case of Indoor office environments and both $30 \mathrm{kHz}$ and $120 \mathrm{kHz}$ subcarrier spacings for UMi Street-canyon and Indoor office environments.

Regarding the mmWave band, it is recommended to use a $120 \mathrm{kHz}$ subcarrier spacing; however, for UMi/UMa O2I and UMa topologies, the interference levels using this numerology are not in acceptable ranges, which is why in these scenarios, it is better to use a $60 \mathrm{kHz}$ spacing subcarrier. Other topologies that allow the use of a $60 \mathrm{kHz}$ subcarrier spacing in mmWave are Indoor office and UMi Street-canyon. Regarding the $15 \mathrm{kHz}$ and $30 \mathrm{kHz}$ subcarrier spacing numerologies, in mmWave, it can be used in any topology (except for RMa, since the channel model used does not allow to scale the DS for frequencies $>6 \mathrm{GHz}$ ), as long as it is at low speeds, i.e., pedestrians moving at an average speed of $3 \mathrm{~km} / \mathrm{h}$.

\section{Conclusions}

Changes in 5G NR numerology have a significant impact on the total system interference, and its influence can be greater or less depending on the system conditions. This has been verified according to the results obtained and the analysis of the ICI and ISI under diverse parameters such as frequency, topology, and UE speed. However, as explained before, increasing subcarrier spacing involves a trade-off between the ICI effect and the ISI effect over the performance of the system. Therefore, by quantifying this interference, it has been possible to determine the best numerology for each of the analysed scenarios within the landscape of 5G.

From the results obtained, it can be concluded that the operating frequency should not be the only parameter to take into account when deciding which numerology should be used in a given system. All 5G OFDM numerologies can be used throughout the $5 \mathrm{G}$ spectrum, depending on the characteristics of the system. Thus, contrary to what was recommended, $120 \mathrm{kHz}$ is not necessarily the best option for mmWave, since the trade-off between the ICI effect and the
ISI effect is not better than the obtained with a $60 \mathrm{kHz}$ subcarrier spacing.

Future work includes analysis adding mobility to the base station, so that the UE is not the only element with a travel speed. This could be useful to determine the best $5 \mathrm{G}$ OFDM numerology for systems where base stations are drones that constantly change height and position, in order to maximize coverage, reaching even sectors with difficult access.

\section{Data Availability}

The datasets generated and analysed during the current study are available in the figshare repository (https://doi.org/ 10.6084/m9.figshare.13067060.v1).

\section{Conflicts of Interest}

The authors declare that there are no conflicts of interest regarding the publication of this paper.

\section{References}

[1] 3GPP, "Study on New Radio (NR) access technology (release 14)," Technical Report 38.912 v14.0.0, March 2017.

[2] A. Osseiran, J. F. Monserrat, and P. Marsch, 5G Mobile and Wireless Communications Technology, Cambridge University Press, Cambridge, UK, 2016.

[3] 3GPP, "Cellular system support for ultra-low complexity and low throughput Internet of things (Ciot)," Technical Report 45.820 v13.1.0, December 2015.

[4] I. Da Silva, S. E. El Ayoubi, O. M. Boldi et al., 5G RAN Architecture and Functional Design, METIS II White Paper, 2016.

[5] F. Schaich and T. Wild, "Waveform contenders for 5G: OFDM vs. FBMC vs. UFMC," in Proceedings of the 6th International Symposium on Communications, Control and Signal Processing (ISCCSP), pp. 457-460, Athens, Greece, May 2014.

[6] A. Aminjavaheri, A. Farhang, A. RezazadehReyhani et al., "Impact of timing and frequency offsets on Multicarrier Waveform candidates for 5G," in Proceedings of the IEEE Signal Processing and Signal Processing Education Workshop (SP/SPE), pp. 178-183, Salt Lake City, UT, USA, 2015.

[7] R. Gerzaguet, N. Bartzoudis, L. G. Baltar et al., "The 5G candidate waveform race: a comparison of complexity and performance," EURASIP Journal on Wireless Communications and Networking, vol. 2017, no. 1, p. 13, 2017.

[8] J. Vihriälä, A. A. Zaidi, V. Venkatasubramanian et al., "Numerology and frame structure for 5G Radio access," in Proceedings of the IEEE 27th Annual International Symposium on Personal, Indoor, and Mobile Radio Communications (PIMRC), pp. 1-5, Valencia, Spain, September 2016.

[9] A. Yazar and H. s. Arslan, "Flexible multi-numerology systems for 5G New Radio," Journal of Mobile Multimedia, vol. 14, no. 4, pp. 367-394, 2018.

[10] A. F. Demir and H. Arslan, "Inter-numerology interference management with adaptive guards: a cross-layer approach," IEEE Access, vol. 8, pp. 30378-30386, 2020.

[11] A. B. Kihero, M. S. J. Solaija, and H. Arslan, "Inter-numerology interference for beyond 5G," IEEE Access, vol. 7, pp. 146512-146523, 2019. 
[12] A. Yazar and H. Arslan, "Reliability enhancement in multinumerology-based 5G New Radio using ini-aware scheduling," EURASIP Journal on Wireless Communications and Networking, vol. 2019, no. 1, p. 110, 2019.

[13] S. Chen and C. Zhu, "ICI and ISI analysis and mitigation for OFDM systems with insufficient cyclic prefix in time-varying channels," IEEE Transactions on Consumer Electronics, vol. 50, no. 1, pp. 78-83, 2004.

[14] D. Tse and P. Viswanath, Fundamentals of Wireless Communication, Cambridge University Press, Cambridge, UK, 2005.

[15] T. Wang, J. G. Proakis, E. Masry et al., "Performance degradation of OFDM systems due to Doppler spreading," IEEE Transactions on Wireless Communications, vol. 5, no. 6, pp. 1422-1432, 2006.

[16] 3GPP, "Technical specification group Radio Access Network; channel model for frequency spectrum above $6 \mathrm{GHz}$ (release 14)," Tech. Rep. 38.900 v14.2.0, December 2016.

[17] 3GPP, "Way forward on further evaluation assumptions for NR waveform," TDoc. R1-165759, August 2016.

[18] G. W. Paper, 5GCM White Paper for Bands up to $100 \mathrm{GHz}$, http://www.5gworkshops.com/5GCM.html, 2015. 